\title{
Makine Öğrenmesi Yöntemlerinde Yüksek Başarım için Kuantum Bilgisayar ve Hesaplamanın Kullanımı
}

\author{
The Usage of Quantum Computer and Computing \\ for High Performance in Machine Learning Methods
}

\author{
Hasan YETIŞ \\ Fırat Üniversitesi, Mühendislik Fakültesi \\ Bilgisayar Mühendisliği \\ h.yetis@firat.edu.tr \\ ORCID: 0000-0001-7608-3293
}

\author{
Mehmet KARAKÖSE \\ Fırat Üniversitesi, Mühendislik Fakültesi \\ Bilgisayar Mühendisliği \\ mkarakose@firat.edu.tr \\ ORCID: 0000-0002-3276-3788
}

\section{Öz}

Günümüzde yüksek hesaplama gücü gerektiren makine öğrenmesi yöntemlerinin artmakta olan performans ihtiyaçlarını karşılamak için çok çekirdekli, çok işlemcili ve grafik işlemcili bilgisayarlar kullanilmaktadır. Bu teknolojiler yöntemlerin hızlanmasını sağlasa da, pratik bir şekilde gerçekleştirilip geliştirilmesi için yeterli değillerdir. Bu çalışmada henüz laboratuvar ortamında uygulamalar başlanan kuantum bilgisayarların ve kuantum hesaplama yöntemlerinin makine ögrenmesi yöntemleri üzerinde mevcut ve potansiyel kullanimlar ele alınmaktadır. Kuantum bilgisayar ve hesaplamanın makine öğrenmesi yöntemlerinde kullanımı, kuantum uyarlamalı algoritmalar, melez kuantum algoritmalar ve kuantum algoritmalar olmak üzere üç başlıkta ele alınmıştır. Günümüzde kuantum hesaplamanın avantajlarından yararlanarak mevcut yöntemlerin iyileştirmesini sağlayan kuantum uyarlamalı algoritmalar yaygın olarak uygulanmaktadır. Alt iş parçacıklarının kuantum bilgisayarlara yaptırılmasını amaçlayan melez kuantum sistemler ile hizlanmalar elde edilmektedir. Tamamen kuantum bilgisayarlarda çalışan makine öğrenmesi yöntemleri için ise kuantum bilgisayarların sahip oldukları donanımsal dezavantajların ortadan kaldırlmasi ve daha çok kuantum yöntem geliştirilmesi gerekmektedir. Genel olarak yapılan çalışmalar, kuantum bilgisayarlarin bellek probleminin çözülmesi ile makine öğrenmesi alanında çığır açıcı gelişmeler olacağ görüşündedir.

Anahtar Sözcükler: kuantum hesaplama, kuantum bilgisayar, makine öğrenmesi, yüksek başarım

Gönderme ve kabul tarihi: 23.12.2020 - 22.02.2021

Makale türü: Derleme

\section{Abstract}

Today, multi-core, multi-processor and graphic processor computers are used to meet the increasing performance needs of machine learning methods that require high computational power. Although these technologies enable the methods to accelerate, they are not sufficient to be implemented and developed in a practical way. In this study, the current and potential uses of quantum computing methods and quantum computers, which have just started to be applied in the laboratory environment, on machine learning methods are discussed. Quantum computing and its use in machine learning methods are discussed under three headings: quantum-inspired algorithms, hybrid quantum algorithms and quantum algorithms. Nowadays, quantum-inspired algorithms are widely applied that enable improvement of existing methods by taking advantage of quantum computing. Accelerations are achieved with hybrid quantum systems that have sub-threads made by quantum computers. For machine learning methods working entirely on quantum computers, it is necessary to eliminate the hardware disadvantages of quantum computers and to develop more quantumbased methods. In general, studies are of the opinion that by solving the memory problem of quantum computers, there will be ground-breaking developments in the field of machine learning.

Keywords: quantum computing, quantum computer, machine learning, high performance.

\section{Giriş}

Teorik olarak geliştirilen algoritmaların uygulanması için gereken kaynak ihtiyacı gün geçtikçe artmaktadır. Yazılım alanında yapılan çalışmalar, daha çok geliştirilen yöntemlerin uygulanması için gereken 
donanımsal kaynağın yetersizliğine takılmaktadır. Örneğin derin öğrenme ile ilgili çalışmalar 1965 yılından itibaren gerçekleşmekteyken, bu alanda pratik anlamda uygulanabilir sistemlerin elde edilmesi yakın geçmişe dayanmaktadır [1]. Günümüze kadar kaynak artışı, yine 1965 yılında ortaya atılan Moore görüşüne göre gerçekleşmekteydi. $\mathrm{Bu}$ görüşe göre, her iki yılda bir tranzistör boyutlarının yarıya inmesi ile performansı yaklaşık olarak ikiye katlanan işlemcilerin elde edileceği savunulmaktadır. Yaklaşık yarım asırdır doğruluğunu ispatlayan bu görüş, tranzistör boyutunda küçülmenin sınırlarına ulaşılması ile yavaş yavaş geçerliliğini yitirmektedir [2].

Makine öğrenmesi gibi üst seviye yapay zekâ uygulamaları genellikle çok sayıda veriye ihtiyaç duymaktadır. Büyük veri kullanımı, önişleme adımlarının fazlalığı veya önişleme adımlarının otomatik olarak gerçekleştirilmesi yapay zekâ yöntemlerinde kaynak ihtiyacı gerektiren faktörlerdir. $\mathrm{Bu}$ kaynak ihtiyacının karşılanabilmesi adına çok çekirdekli, çok işlemcili ve grafik işlemcili bilgisayarlar kullanılmaktadır [3]. Makine öğrenmesi yöntemlerinin duyduğu performans ihtiyacı bahsi geçen teknolojilerle kısmen karşılansa da günümüzde yüksek başarım ile çalışan makine öğrenmesi yöntemlerinin kuantum bilgisayarlar ile mümkün olacağı görüşü baskındır. Ancak bunun için bellek birimi olmaması gibi dezavantajların ortadan kalkması gerekmektedir [4-6]. Klasik işlemcili bilgisayar, grafik işlemcili bilgisayar, çok çekirdekli ve işlemcili süper bilgisayar ve kuantum bilgisayarların performans karşılaştırmaları Çizelge-1 ile verilmiştir. Çizelgede yer alan süper bilgisayar 500 metrekarelik bir alanda 26 çekirdekli 41.000 işlemcinin birleştirilmesi ile oluşturulmuştur. $\mathrm{Bu}$ tarz süper bilgisayarlar onlarca MW'l1k enerji harcamaktayken, kuantum bilgisayar sadece 25 kW'lık enerji harcamaktadır $[7,8]$. Bu da kuantum bilgisayarların ihtiyaç duyulan yüksek başarım ihtiyacinı, süper bilgisayarlardan binlerce kat fazla performans ve yüzlerce kat düşük enerji ile karşıllama potansiyelini göz önüne sermektedir. Klasik bilgisayarlar ile kuantum bilgisayarlar arasındaki farklar Çizelge-2 ile verilmiştir.
Çizelge-1: Klasik işlemci, grafik işlemci, süper bilgisayar ve kuantum bilgisayar karşılaştırması $[7,8]$

\begin{tabular}{|l|c|c|c|}
\hline $\begin{array}{l}\text { Birim } \\
\text { (Hız x Çekirdek) }\end{array}$ & $\begin{array}{c}\text { En çok } \\
\text { işlem sayısı } \\
/ \text { s }\end{array}$ & $\begin{array}{l}\text { Geçen } \\
\text { Süre* }\end{array}$ & Güç \\
\hline $\begin{array}{l}\text { Klasik İşlemci } \\
(3,4 \mathrm{GHz} \times 4)\end{array}$ & $\begin{array}{l}13,6 \text { milyar } \\
\left(13,6 \times 10^{9}\right)\end{array}$ & $780 \mathrm{y} 1 \mathrm{l}$ & $\sim 65 \mathrm{~W}$ \\
\hline $\begin{array}{l}\text { Grafik İşlemci } \\
(1.7 \mathrm{GHz} \times 2500)\end{array}$ & $\begin{array}{c}4,25 \text { trilyon } \\
\left(4,25 \times 10^{12}\right)\end{array}$ & $2,5 \mathrm{y} 11$ & $\sim 300 \mathrm{~W}$ \\
\hline $\begin{array}{l}\text { Süper Bilgisayar } \\
\text { (9 GHz x 10,7 } \\
\text { milyon) }\end{array}$ & $\begin{array}{c}100 \\
\text { kuadrilyon } \\
\left(100 \times 10^{15}\right)\end{array}$ & 1 saat & $\sim 20 \mathrm{M}$ \\
\hline $\begin{array}{l}\text { Kuantum } \\
\text { Bilgisayar } \\
\text { (50 kübit) }\end{array}$ & - & 1 saniye & $\sim 25 \mathrm{~kW}$ \\
\hline
\end{tabular}

* Aynı işin tamamlanması için geçen süre.

Çizelge-2: İkili ve Kuantum Bilgisayar karşılaştırması $[7,8]$

\begin{tabular}{|c|c|c|}
\hline & İkili Bilgisayar & $\begin{array}{l}\text { Kuantum } \\
\text { Bilgisayar }\end{array}$ \\
\hline İșlem birimi & Bit & Kübit \\
\hline Uygulama & Lojik sinyaller & $\begin{array}{l}\text { Foton veya atom } \\
\text { altı parçacıklar }\end{array}$ \\
\hline Determinizm & Kesindir & Olasılıksaldır \\
\hline Hesaplama & $\begin{array}{l}\text { İkili gösterim ve } \\
\text { Boolean cebri }\end{array}$ & $\begin{array}{l}\text { Bra-ket gösterimi } \\
\text { ve Matris cebri }\end{array}$ \\
\hline $\begin{array}{l}\text { Çalışma } \\
\text { Koșulları }\end{array}$ & $\begin{array}{l}\text { Günlük sıcaklık } \\
\text { değerleri }\end{array}$ & $0^{\circ} \mathrm{K}\left(-273,3^{\circ} \mathrm{C}\right)$ \\
\hline $\begin{array}{l}\text { Kapasite } \\
\text { (Günümüz) }\end{array}$ & $\begin{array}{l}\approx 400 \quad \text { milyon } \\
\text { tranzistör }\end{array}$ & $\begin{array}{l}\text { IBM-Q: } 53 ; \quad \text { D- } \\
\text { Wave: } 2000 \text { kübit }\end{array}$ \\
\hline Modelleme & Turing makinesi & Kuantum devreleri \\
\hline
\end{tabular}

Avantajları yukardaki gibi sayılan kuantum bilgisayarların gerçekleştirilmesinin önünde birçok zorluk da yer almaktadır. Bu zorluklar aşağıdaki gibi listelenebilir $[9,10]$ :

- Yüksek hızlı hesaplama yapıldığından veri akıșının kontrol edilmesi daha zordur.

- Atom altı parçacıkların kararlı çalışması için ortamın Mutlak $0\left(-273.3^{\circ} \mathrm{C}\right)^{\prime} \mathrm{da}$ tutulması gerekliliği.

- Kübitlerin kararlı yapıda uzun süre tutulmasının mümkün olmaması. Dolayısı ile bellek olmamas1.

- Çalışma koşullarının ideali yakalamaması sebepleri ile hatalı sonuçların çıkabilmesi.

- Kuantum bilgisayarlarda çalışabilmesi için yeterli sayıda mevcut kuantum algoritmanın bulunmamas1. 
Günümüzde avantajlarının yanında dezavantajlara da sahip kuantum bilgisayarların, dezavantajlarının ortadan kaldırılması ile makine öğrenmesi yöntemleri için çığır açıcı olması beklenmektedir $[4,6]$.

\section{Kuantum Hesaplama}

Kuantum hesaplama kuantum terimlerinin ve mekaniklerinin hesaplama ișlemlerinde kullanılmana verilen genel isimdir. Süper pozisyon ve dolanıklık bu amaçla sıklıkla kullanılan kuantum hesaplama terimleridir $[11,12]$. Süper pozisyon kavram1, kuantum dünyasında bir parçacığın gözlemlenene kadar durumunun belli olmaması durumudur. Ancak gözlemlenen parçacıklar çökme işlemine tabi tutularak 0 veya 1 durumuna geçerler [13]. Kübitler matematiksel olarak Dirac (bra-ket) notasyonu ile gösterilmektedir. $c_{0}{ }^{2}$ gözlem sonucunun 0 olma olasılığ1 ve $c_{1}^{2} 1$ olma olasılığı olmak üzere, Dirac notasyonu gösterimi (1) ve (2)'de verildiği gibidir [14]. Atom altı parçacıkların dönüş yönlerinin geometrik olarak gösterimi ise Bloch dairesi ile gösterilir. Bloch dairesi ve Dirac notasyonu karşılıklarının gösterimi Şekil-1 ile verilmiştir [15].

$|\psi\rangle=\left[\begin{array}{l}c_{0} \\ c_{1}\end{array}\right] \quad \rightarrow|0\rangle=\left[\begin{array}{l}1 \\ n\end{array}\right], \quad|1\rangle=\left[\begin{array}{l}0 \\ 1\end{array}\right]$
$|\psi\rangle=c_{0} \cdot|0\rangle+c_{1} .|1\rangle, \quad c_{0}{ }^{2}+c_{1}{ }^{2}=1$

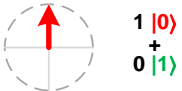

(a)

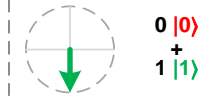

(b)

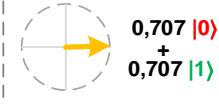

(c)
Şekil-1: Kübitlerin Bloch dairesi ve Dirac notasyonu ile gösterimi (a) $|0\rangle$ gösterimi, (b) $|1\rangle$ gösterimi, (c) $|+\rangle$ gösterimi.

Kübitler üzerinde işlemler kuantum kapılar aracılığıyla gerçekleşir. Şekil-2'de Pauli-X kapısının, ya da başka bir deyişle değil kapısının uygulanması ve sonucun gözlemlenmesi ile elde edilecek durumların olasılıkları gösterilmiştir. Kuantum kapılar tek kübite uygulanabileceği gibi birden fazla kübite uygulanan kapılar da bulunmaktadır. Kuantum bilgisayarların bütün değerler için paralel işlem yapması, girişlerin Şekil-1.c'deki gibi süper pozisyon halinde verilmesi sonucunda gerçekleşir. Sonuçta hem 0 ile hem 1 ile işlem yapılması halinde çıkacak sonuçlar olasılıksal olarak hesaplanır. $\mathrm{Bu}$ durum gerçek kuantum bilgisayarlarda, sonucun olasılıksal olarak bir durumda görülmesi ile gerçekleşir. Kuantum bilgisayarlardaki sonuçlar olasılıksal olduğundan, algoritmalar aynı girişler için birden fazla kez çalıştırılarak sonuçların nerde yoğunlaştığına bakılır. Genellikle kuantum algoritmalar 1024, 2048, 4096 veya $8192 \mathrm{kez}$ çalıştırılır.

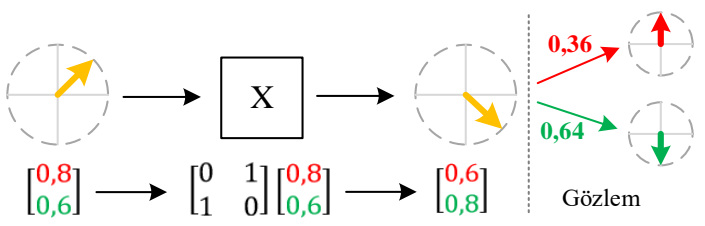

Şekil-2: Pauli-X kapısının uygulamasının Bloch dairesi ve Dirac notasyonu üzerinde gösterimi.

Literatürde tek kübitlik ve çok kübitlik birçok kuantum kap1 yer almaktadır. Bunların sik kullanılanlarından bazıları Çizelge-3 ile verilmiştir [16]. Kuantum devreler bu kapıların ardışık olarak kullanılması ile oluşturulurlar. Bir kapıyı kübit sayısını artırarak kullanmak için kapının kendisi ile tensör çarpımı gerçekleştirilir. Tensör çarpımı (3) ile gösterildiği şekilde gerçekleşmektedir [17].

$\left[\begin{array}{ll}a & b \\ c & d\end{array}\right] \otimes\left[\begin{array}{ll}x & y \\ z & t\end{array}\right]=\left[\begin{array}{llll}a x & a y & b x & b y \\ a z & \text { at } & b z & b t \\ c x & c y & d x & d y \\ c z & c t & d z & d t\end{array}\right]$ 
Çizelge-3: Sık kullanılan kuantum kapılardan bazıları [16]

\begin{tabular}{|c|c|c|c|c|c|c|c|c|}
\hline Kapı Adı & $\begin{array}{c}\text { Sembo } \\
\text { lü }\end{array}$ & \multicolumn{7}{|c|}{ Matris Formu } \\
\hline Hadamard & $\mathrm{H}-$ & \multicolumn{7}{|c|}{$\frac{1}{\sqrt{2}}\left[\begin{array}{cc}1 & 1 \\
1 & -1\end{array}\right]$} \\
\hline Pauli-X & $X$ & \multicolumn{7}{|c|}{$\left[\begin{array}{ll}0 & 1 \\
1 & 0\end{array}\right]$} \\
\hline Pauli-Y & Y & \multicolumn{7}{|c|}{$\left[\begin{array}{cc}0 & -i \\
i & 0\end{array}\right]$} \\
\hline Pauli-Z & Z & \multicolumn{7}{|c|}{$\left[\begin{array}{cc}1 & 0 \\
0 & -1\end{array}\right]$} \\
\hline $\mathrm{Faz}$ & $\mathrm{S}$ & \multicolumn{7}{|c|}{$\left[\begin{array}{ll}1 & 0 \\
0 & i\end{array}\right]$} \\
\hline$\pi / 8$ & $\mathrm{~T}$ & \multicolumn{7}{|c|}{$\left[\begin{array}{cc}1 & 0 \\
0 & e^{i \pi / 4}\end{array}\right]$} \\
\hline $\begin{array}{c}\text { C-NOT } \\
\text { (controlled- } \\
\text { NOT) } \\
\text { (2 kübit) }\end{array}$ & & & $\begin{array}{l}1 \\
0 \\
0 \\
0\end{array}$ & $\begin{array}{l}0 \\
1 \\
0 \\
0\end{array}$ & $\begin{array}{l}0 \\
0 \\
0 \\
1\end{array}$ & & & \\
\hline $\begin{array}{c}\text { Swap } \\
\text { (2 kübit) }\end{array}$ & & & $\begin{array}{l}1 \\
0 \\
0 \\
0\end{array}$ & $\begin{array}{l}0 \\
0 \\
1 \\
0\end{array}$ & $\begin{array}{l}0 \\
1 \\
0 \\
0\end{array}$ & & & \\
\hline $\begin{array}{l}\text { Toffoli } \\
\text { (3 kübit) }\end{array}$ & 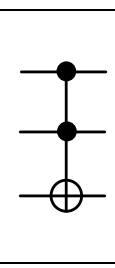 & {$\left[\begin{array}{ll}1 & 0 \\
0 & 1 \\
0 & 0 \\
0 & 0 \\
0 & 0 \\
0 & 0 \\
0 & 0 \\
0 & 0\end{array}\right.$} & $\begin{array}{l}0 \\
0 \\
1 \\
0 \\
0 \\
0 \\
0 \\
0\end{array}$ & $\begin{array}{l}0 \\
0 \\
0 \\
1 \\
0 \\
0 \\
0 \\
0\end{array}$ & $\begin{array}{l}0 \\
0 \\
0 \\
0 \\
0 \\
1 \\
0 \\
0 \\
0\end{array}$ & $\begin{array}{l}0 \\
0 \\
0 \\
0 \\
0 \\
0 \\
1 \\
0 \\
0\end{array}$ & $\begin{array}{l}0 \\
0 \\
0 \\
0 \\
0 \\
0 \\
0 \\
1\end{array}$ & $\left.\begin{array}{l}0 \\
0 \\
0 \\
0 \\
0 \\
0 \\
1 \\
0\end{array}\right]$ \\
\hline
\end{tabular}

Sık kullanılan kapıların yanında özelleştirilmiş kapılar elde etmek de mümkündür. Bir kübitin $\Delta \theta$ açısıyla dönmesini sağlayacak kuantum kap1 denklemi (4)'te verildiği gibi hesaplanır.

$U(\Delta \theta)=\left[\begin{array}{ll}\cos (\Delta \theta) & -\sin (\Delta \theta) \\ \sin (\Delta \theta) & \cos (\Delta \theta)\end{array}\right]$

Klasik ikili devrelerde Boolean cebrine göre gerçekleştirilen işlemlerin kuantum karşılıkları da geliştirilmektedir. Örneğin kuantum CNOT kapısı ile ikili XOR kapısının doğruluk tablosu incelendiğinde aynı işlevi gördükleri anlaşılmaktadır. Tam toplayıcı devre tasarımının mantık kapıları ve kuantum kapılar ile gerçekleştirilmesi Şekil-3 ile verilmiştir.
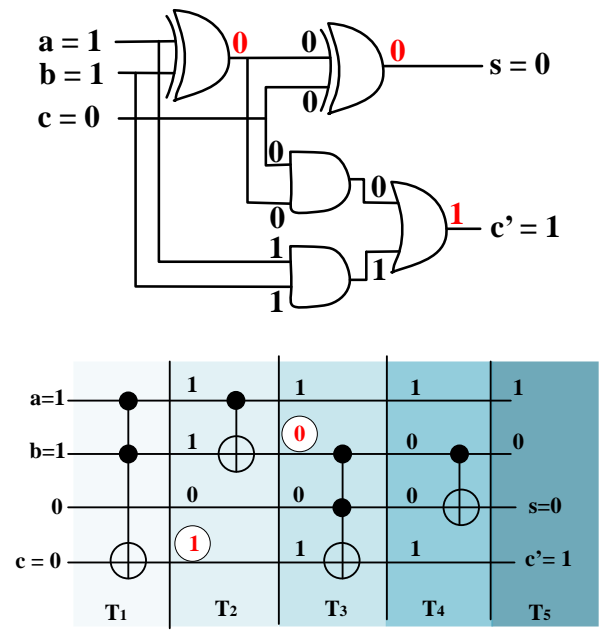

Şekil-3: Tam toplayıcı devreler (a) Klasik devre, (b) Kuantum devre.

\section{Makine Öğrenmesi Yöntemlerinde Kuantum Hesaplama ve Kuantum Bilgisayarların Kullanımı}

Kuantum mekaniklerinin makine öğrenmesi yöntemlerinde kullanımları temel olarak adyabatik tabanlı çalıșmalar ve kuantum kapı tabanlı çalıșmalar olarak ikiye ayrılabilir. Adyabatik kuantum sistemler, belirli bir graf yapısına göre bağlanan parçacıkların Hamilton denklemine göre düşük enerjili duruma geçmesine dayanır $[18,19]$. Bu şekilde kuantum tavlama ad1 verilen optimizasyon yöntemi uygulanmış olur [20]. Kuantum bilgisayar çalışmalarının başını çeken D-Wave Sistemler adyabatik tabanlı çalıșmalar yürütürken, IBM-Q kuantum bilgisayarları kuantum kapı tabanlı çalışmalar üzerine yoğunlaşmaktadır [21, 22].

Literatürde kapı tabanlı kuantum hesaplama ile ilgili gerçekleştirilen çalışmalar genellikle üç başlık altında incelenebilir. Bunlar kuantum verilerin klasik algoritmalarda kullanıldığı kuantum uyarlamalı algoritmalar, alt iş parçacıklarının kuantum bilgisayarlara yaptırıldığı melez kuantum algoritmalar ve tamamen kuantum bilgisayarlar üzerinde çalışan kuantum algoritmalardır $[6,14]$. Kuantum uyarlamalı algoritmalar kuantum verilerin klasik bilgisayarlarda işlenmesiyle, melez kuantum algoritmalar yine kuantum verilerin bazılarının kuantum bazılarının klasik bilgisayarda işlenmesiyle, kuantum algoritmalar ise bu verilerin tamamen kuantum 
bilgisayarlarda işlenmesi ile çalışır. Şekil-4'te kuantum uyarlamalı, melez kuantum ve kuantum algoritmalar verilmiştir. Her üç başlık için de literatürde birçok çalışma yer almaktadır. Kuantum uyarlamalı algoritmalar ile her ne kadar kuantum bilgisayarların gerçek hesaplama potansiyeli kullanılamasa da, kuantum hesaplamanın sağlamış oldukları avantajlar sayesinde algoritmaların orijinal versiyonlarına göre çözüme daha yakın veya nispeten daha kısa sürede sonuçlar elde edilmektedir. $\mathrm{Bu}$ algoritmalar, kuantum algoritmaların geliştirilmesi için geçiş görevi görmektedir.

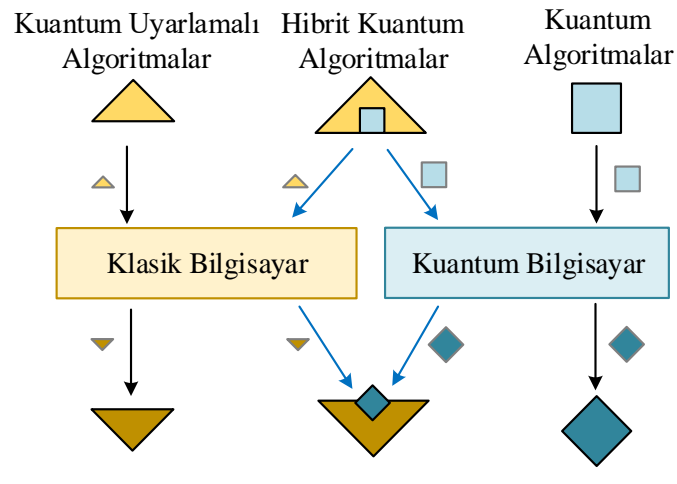

Şekil-4: Kuantum hesaplamanın makine öğrenmesi yöntemlerinde kullanılması [6].

\subsection{Kuantum Uyarlamalı Algoritmalar}

Yüksek hesaplama gücüne ihtiyaç duyan makine öğrenmesi yöntemlerinin kuantum versiyonlarının uygulamasının önündeki engeller nedeniyle, kuantum uyarlamalı yöntemler ortaya atılmıştır. Özellikle son y1llarda birçok meta sezgisel yöntemin kuantum uyarlamalı versiyonları üzerine çalışmalar gerçekleştirilmiştir. Kübitlerin kullanımı ile geliştirilen Kuantum Uyarlamalı Evrimsel Algoritmalar, Kuantum Uyarlamalı Genetik Algoritmalar, Kuantum Uyarlamalı Parçacık Sürü Optimizasyonu, Kuantum Uyarlamalı Diferansiyel Gelişim Algoritmaları, Kuantum Uyarlamalı Yapay Ar1 Kolonisi Algoritmaları, Kuantum Uyarlamalı Karınca Koloni Algoritmaları, Kuantum Uyarlamalı Ateş Böceği Algoritmaları bunlara örnek olarak verilebilir [23, 24]. Yapılan çalışmaların birçoğu algoritmaların yakınsama hızını artırmaya yönelik olmakla birlikte, zaman karmaşıklığını azaltmak adına yapılan çalışmalar daha azdır [24].
Kuantum uyarlamalı algoritmaların kullanımı meta sezgisel algoritmalar ile sınırlı kalmamaktadır. Birçok algoritma için kuantum hesaplama terimlerini kullanılarak, çeşitli avantajlar elde edilmeye çalışılmıştır. Bu konuyla ilgili özellikle son yıllarda gerçekleşen çalışmalar Çizelge-4 ile özetlenmiştir. Çalışmalar incelendiği zaman, genellikle kübit ve kuantum kapı gibi yapıların klasik algoritmalara uyarlanması ile başarımda artış elde edildiği görülmektedir.

\section{Çizelge-4: Son yıllarda kuantum uyarlamalı makine öğrenmesi alanında gerçekleştirilen belli başlı çalışmalar.}

\begin{tabular}{|c|c|c|}
\hline Yil & Gerçekleştirilen Çalışma & \\
\hline $\begin{array}{l}\text { Erken } \\
\text { Erişim }\end{array}$ & $\begin{array}{lr}\text { Yapay Sinir Ağlarına } & \text { (YSA), } \\
\text { bulanık küme ve kuantum } \\
\text { hesaplama terimleri ile yeni bir } \\
\text { yorum getirerek } & \text { ikili } \\
\text { sınıflandırma } & \text { işlemi } \\
\text { gerçekleştirmiştir. }\end{array}$ & [25] \\
\hline 2019 & $\begin{array}{l}\text { Kerenidis ve Prakash [26] } \\
\text { tarafindan ortaya atılan kuantum } \\
\text { öneri algoritmasından esinlenerek } \\
\text { kuantum uyarlamalı öneri } \\
\text { algoritmasını uygulamıştır. }\end{array}$ & [27] \\
\hline 2018 & $\begin{array}{l}\text { YSA'nın gerçekleştirilmesinde } \\
\text { kübitler ve kuantum kapıları } \\
\text { kullanmış ve bu sayede başarımın } \\
\text { arttığı sonucuna varmıştır. }\end{array}$ & [28] \\
\hline 2015 & $\begin{array}{lr}\text { Kuantum Derin } & \text { Öğrenme } \\
\text { Algoritmasından } & {[29]} \\
\text { uyarlayarak Boltzman } & \text { Makineleri } \\
\text { için Eğitim süreci önermiştir. }\end{array}$ & [30] \\
\hline 2010 & $\begin{array}{lcr}\text { Kuantum } & \text { Uyarlamalı Derin } \\
\text { Öğrenme } & \text { Ağı önermiştir. } \\
\text { Önerilen yöntemin klasik } \\
\text { yönteme göre daha az hata ile } \\
\text { çalıștığı sonucuna varmıştır. }\end{array}$ & [31] \\
\hline
\end{tabular}

\subsection{Melez Kuantum Algoritmalar}

Makine öğrenmesi yöntemlerinin gerçek kuantum hesaplama avantajlarından yararlanması için, klasik ile kuantum bilgisayarların melez olarak kullanıldığı 
çalışmalar yapılmaktadır [32]. $\mathrm{Bu}$ çalışmalarda kuantum bilgisayarlar, bilinen alt problemlere indirgeme yöntemi ile karmaşı algoritmaların daha basit bölümlerini çalıştırır ve bu sayede kısmen de olsa bir hız artışı elde edilir. Yaygın olarak bilinen kuantum algoritmalar arasinda Deutsch Shor, Grover, Swap Test, QSVE (Prakash-Kerenidis) ve HHL (Harrow, Hassidim and Lloyd) Algoritmaları yer almaktadır [5, 6, 33]. Deutsch Algoritması temel olarak fonksiyonun sabit olup olmadığının tespit etmede, Shor Algoritması sayıları asal çarpanlara ayırmada, Grover Algoritması arama işleminde, Swap Test iki kuantum durumun birbirinden ne kadar farklılaşabileceğini araştırmada, QSVE ve HHL ise lineer denklem çözümünde kullanılan algoritmalardır. Kuantum makine öğrenmesi yöntemlerinin incelendiği bir çalışmada Destek Vektör Makineleri (DVM), K-Ortalama, Temel Bileşen Analizi (PCA) ve Lineer Diskriminant Analizi (LDA) için kuantum alt programların uygulanması ile hız artışı olduğu rapor edilmiştir [32]. Sonuçlar Çizelge-5'te verilmiştir.

Çizelge-5: Melez kuantum algoritmalar ve sağladıkları hız artışları [33].

\begin{tabular}{|l|l|l|}
\hline \multicolumn{1}{|c|}{ Algoritma } & Alt Program & Hız Artışı \\
\hline DVM & HHL & Eksponansiyel \\
\hline K-Ortalama & $\begin{array}{l}\text { Swap Test, } \\
\text { Grover }\end{array}$ & Kuadratik \\
\hline PCA & HHL & Eksponansiyel \\
\hline LDA & HHL & Eksponansiyel \\
\hline
\end{tabular}

Melez kuantum alanında yapılan bir çalışmada ise Sınırlı Boltzman Makinesinin (LBM) kuantum bilgisayarlarda, Konvolüsyonel Sinir Ağlarının (CNN) yüksek hesaplama gücüne sahip bilgisayarlarda, Konuşan Sinir Ağlarının (SNN) ise Nöromorfik bilgisayarlarda çalıştırılarak üç farklı mimariye sahip bilgisayarın melez kullanımı amaçlanmıştır [34]. Bir başka çalışmada ise gözetimli, gözetimsiz ve takviyeli öğrenmede alt program olarak kullanılması için kuantum yöntemler önerilmiştir [35]. Beneditti ise çalışmasında kuantumdan yararlanarak geliştirdiği Helmholtz makinesinin derin ögrenme için melez kullanılabileceğini savunmuştur [36].

\subsection{Kuantum Algoritmalar}

Günümüzde tam anlamıyla kuantum bilgisayarlarda çalışacak makine öğrenmesi algoritmaları oldukça sınırlıdır. Kuantum dolanıklık kavramının kullanılması ile farklı bir hesaplama yaklaşımı olan kuantum tavlama yöntemi D-Wave sistemler tarafindan uygulanmaktadır [22]. Diğer taraftan Google ve Nasa gibi şirketlerin öncülüğünde QuAIL araştırma grubu tarafindan kuantum yapay zekâ teknikleri üzerine araştırmalar yürütülmektedir [37]. Kuantum kapı temelli çalışmalara bakıldığında gerçekleştirilen çalışmalar arasında en basit YSA olarak bilinen perseptron modeli uygulaması yer almaktadır [38-40]. Tacchino vd. yaptıkları çalışmada $2 \times 2$ piksellik siyah beyaz görüntüleri serileştirilerek 4 kübit ile ifade edilmiştir. Ardından siyah pikseller 0, beyaz pikseller 1 olmak üzere tasarlanan kuantum devreye verilmiştir. Sonuçta basit olarak $2 \times 2$ 'lik alanda çizgi formunda olan ve olmayan görseller ayırt edilmiştir. Gerçekleştirilen çalışmada oluşturulan 4 kübitlik kuantum perseptron devresi Şekil-5 ile verilmiştir [40].

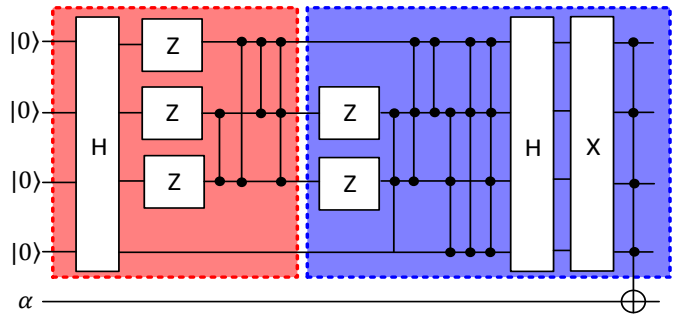

Şekil-5: Tacchino vd. tarafından oluşturulan 4 kübitlik perseptron kuantum devre modeli [40].

Kuantum makine öğrenmesi alanındaki çalışmalar, ihtiyaç duydukları işlem gücünün kuantum bilgisayarlarca karşılanabileceği düşünüldüğünden, genellikle YSA ve derin öğrenme üzerine yoğunlaşmaktadır. Çizelge-6'da bu alanda yapılan çalışmalar özetlenmiştir. 


\section{Çizelge-6: Son yıllarda kuantum makine öğrenmesi alanında gerçekleştirilen belli başı çalışmalar.}

\begin{tabular}{|c|c|c|}
\hline Yll & Gerçekleştirilen Çalışma & \\
\hline 2019 & $\begin{array}{l}\text { Kuantum foton işlemciler için, } \\
\text { sinir ağları özelliklerini kuantum } \\
\text { optikal domenine dönüştürmek } \\
\text { için yap } 1 \text { önermiştir. }\end{array}$ & [41] \\
\hline 2018 & 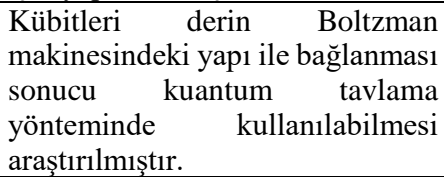 & [19] \\
\hline 2018 & $\begin{array}{l}\text { Kuantum Boltzman Makinesi } \\
\text { geliştirilmiştir. Kuantum tavlama } \\
\text { yöntemi için uygulanması durumu } \\
\text { incelenmiştir. } \\
\text { sistemlere, ufak değişiklikler gile } \\
\text { uygulanabilir olduğu rapor } \\
\text { edilmiştir. }\end{array}$ & [42] \\
\hline 2018 & $\begin{array}{l}\text { MINST veri kümesini AlexNet ve } \\
\text { D-Wave üzerinde sinıflandırmış. } \\
\text { D-Wave } 95.5 \% \text {, CNN ise } 94-95 \% \\
\text { arası başarım elde etmiştir. D- } \\
\text { Wave üzerinde MINST veri } \\
\text { kümesini uygulamak için boyut } \\
\text { indirgemiştir. }\end{array}$ & [43] \\
\hline 2018 & $\begin{array}{l}\text { Kuantum sinir ağları modeli ile } \\
\text { simülasyon üzerinden eğitim } \\
\text { işlemi gerçekleştirerek, iki sınıfı } \\
\text { ayırt edilmesi başarılmıştır. Kübit } \\
\text { sinırlamaları kalkınca kuantum } \\
\text { bilgisayarlarda } \\
\text { uygulanabilecektir. }\end{array}$ & [44] \\
\hline 2017 & $\begin{array}{l}\text { Kuantum tavlamanın modelleme } \\
\text { kapasitesini artırılması ve bu } \\
\text { modellerde daha fazla veri } \\
\text { kullanılabilmesi için çalışmalar } \\
\text { yapılmıştır. }\end{array}$ & [45] \\
\hline 2014 & 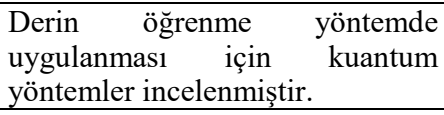 & [46] \\
\hline 2013 & $\begin{array}{l}\text { Kuantum Perseptron sinir ağının } \\
\text { düşük maliyetli öğrenmeyi } \\
\text { başarabileceği yeni bir } \\
\text { hesaplamall yaklaşım sunmuştur } \\
\text { (AQPNN). }\end{array}$ & [47] \\
\hline
\end{tabular}

\section{Makine Öğrenmesi Yöntemlerinde Kuantum Hesaplama ve Kuantum Bilgisayarların Geleceği}

Literatürde mevcut kuantum bilgisayarların makine öğrenmesi yöntemlerinde kullanılması önünde engelleri yok sayarak yapılan çalıșmalar yer almaktadır $[48,49]$. Bunlardan en önemlileri kuantum devrelerin parametrelere bağlanması ve kuantum bellek birimlerinin kullanılabilmesidir. $\mathrm{Bu}$ alanda yapılan araştırmaların birinde, parametre kabul eden kuantum bilgisayarlarda çalışabilmesi için derin öğrenme yönteminin uygulamasında kullanılabilecek yöntemler geliştirilmiştir [48]. Yapılan bir başka çalıșmada ise, kuantum bellek birimine sahip kuantum bilgisayarların gerçekleştirildiği varsayılarak, derin öğrenmede kullanılan havuzlama ve konvolüsyon işlemlerinin kuantum versiyonları üretilmiştir [49].

Her ne kadar kuantum uyarlamalı ve melez kuantum başlığı altında yapılan çalışmalar önemli avantajlar sağlasa da makine öğrenmesinin asıl sıçrayış tamamen kuantum bilgisayarlarda çalışabilen yöntemlerinin elde edilmesi ile gerçekleşecektir. Kuantum bilgisayarlar günümüzde her ne kadar bilgisayar olarak isimlendirilse de henüz yapısı gereği işlemci olmanın ötesine geçememiştir. Kübitlerin gözlemlenmeden bellekte tutulmasını üzerine çalışmalar yürütülürken, günümüzde henüz kuantum ram gibi bellek birimlerinin kullanılması mümkün değildir [9]. $\mathrm{Bu}$ durum kuantum algoritmaların geliştirilmesinin önündeki en büyük dezavantajlardan birini olușturmaktadır. Yakın gelecekte qRAM (kuantum RAM) olarak isimlendirilen belleklerin ortaya çıkması ile bu dezavantajın ortadan kaldırılması öngörülmektedir [5]. Bu alanda yapılan çalışmalar ile varılan kanılar aşağıda listelenmiştir:

- Klasik bilgileri kuantum formunda tutacak qRAM gibi araçların oluşturulması kuantum makine öğrenmesinin önünde aşılması gereken önemli bir engeldir [4].

- qRAM kullanılması ile makine öğrenmesi eğitim aşaması, klasik algoritmalara göre veriye kuadratik olarak daha az erişim sağlayarak gerçekleşebilecektir [4].

- qRAM yapısının geliştirilmesi zor bir problem olsa da, bunun için gereken materyaller doğada bulunmaktadır ve yapıyı elde etmek için açık bir yol bulunmaktadır [4].

- Kuantum bilgisayarların makine öğrenmesinde kullanılması ve çığır açması için qRAM yapısının geliştirilmelidir [4]. 
- qRAM yapısının geliştirilmesi, kuantum makine öğrenmesi alanı için yeni bir kap1 açacaktır [6].

- Kuantum algoritmalardaki gerçek hız kazancı için qRAM yapılarının kullanılması gerekmektedir [6].

\section{Sonuçlar}

$\mathrm{Bu}$ çalışmada makine öğrenmesi yöntemlerinde yüksek başarım elde etmek için kuantum bilgisayar ve hesaplama tekniklerinin kullanılması ele alınmıştır. Makine öğrenmesi alanında kuantum bilgisayar ve hesaplamanın kullanılması üzerine gerçekleştirilen literatür çalışmaları incelenerek mevcut durum analiz edilmiştir. Bu alanda gerçekleştirilen çalışmalar temel olarak kuantum uyarlamalı algoritmalar, melez kuantum algoritmalar ve kuantum algoritmalar olmak üzere üç ana başlıkta ele alınmıştır. Kuantum bilgisayarların uygulamalarındaki zorluklar, kuantum hesaplamanın avantajlarından faydalanmak isteyen araştırmacıları kuantum uyarlamalı algoritmalar geliştirmeye teşvik etmiştir. Kübit ve kuantum kap1 terimlerinin klasik algoritmalara uygulanması ile gerçekleştirilen bu çalışmalar incelendiğinde, genellikle çözüm kalitesi açısından iyileşmeler yakalandığ1 görülmektedir [23-31]. Ancak kuantum hesaplamanın asıl avantajı kuantum bilgisayarların devreye girmesi ile ortaya çıkmaktadır. $\mathrm{Bu}$ avantajlardan kısmen de olsa yararlanmak adına, kuantum bilgisayarların k1smen kullanıldığ kuantum yöntemler uygulanmıştır. $\mathrm{Bu}$ yöntemler ile klasik uygulamalara göre hızlanmalar elde edildiği vurgulanmıştır. Son olarak ise makine öğrenmesi yöntemlerinin kuantum bilgisayarlarda çalışabilmesi adına önerilerde bulunan kuantum yöntemler incelenmiştir. $\mathrm{Bu}$ yöntemler, yüksek başarım açısından kuantum bilgisayarların asıl avantajlarının görüleceği yöntemler olmaktadır. Ancak günümüzde kuantum bilgisayarlar sahip oldukları dezavantajlar sebebiyle henüz işlemci seviyesinde kalmaktadır. Bu alandaki çalışmalar incelendiğinde perseptron gibi basit yapıların kuantum bilgisayarlarca uygulandığ veya daha karmaşık yöntemlerin uygulanması için teorik çalışmaların yapıldığı görülmektedir [18-49]. Diğer taraftan parametrelere bağlanabilen ve kuantum bellek birimi kullanabilen kuantum devrelerin uygulanabilmesi ile kuantum makine öğrenmesi yöntemlerinin önündeki büyük engellerden birinin kalkacağı incelenen çalışmalarca varılan yaygın bir görüştür [4-6]. Özellikle kuantum bellek birimlerinin kullanılabilir olması ile derin öğrenme gibi büyük kaynak ihtiyacı olan yöntemlerin daha hızlı eğitilmesi kuantum bilgisayarlar tarafından sağlanacaktır. Bu yöntemlerin geliştirilmesinin önündeki en büyük engellerden biri olan geçen süre çok daha kısalacağından, kuantum bilgisayarların makine öğrenmesi yöntemlerinde kullanılması ile makine öğrenmesi alanında çığır açılacağı öngörülmektedir.

\section{Kaynakça}

[1] Şeker A., Diri, B. ve Balık, H., Derin Öğrenme Yöntemleri ve Uygulam alar1

Hakkında Bir İnceleme, Gazi Mühendislik Bilimleri Dergisi, 3(3), 47-64, 2017.

[2] Xiu, L., Time Moore: Exploiting Moore's Law From The Perspective of Time, IEEE Solid-State Circuits Magazine, cilt 11, no. 1, pp. 39-55, 2019.

[3] Gates, M., Heath, M. T. ve Lambros, J., Highperformance hybrid CPU and GPU parallel algorithm for digital volume correlation, The International Journal of High Performance Comp. Applications, 29(1), 92-106, 2015.

[4] Biamonte, J., Wittek, P., Pancotti, N., Rebentrost, P., Wiebe N. ve Lloyd, S., Quantum machine learning, Nature, cilt 549, no. 7671, p. 195-202, 2017.

[5] Ciliberto, C., Herbster, M., Ialongo, A. D., Pontil, M., Rocchetto, A., Severini, S. ve Wossnig, L., Quantum machine learning: A classical perspective, Proceedings of the Royal Society A: Mathematical, Physical and Engineering Sciences, cilt 474, no. 2209, 2018.

[6] Adcock, J. , Allen, E., Day, M., Frick, S., Hinchliff, J., Johnson, M., Morley-Short, S., Pallister, S., Price, A. ve Stanisic, S., Advances in quantum machine learning, arXiv preprint arXiv:1512.02900v1, 2015.

[7] ACM, Super Computing's Super Energy Needs, and What to Do About Them, Url: https://cacm.acm.org/news/192296-supercomp utings-super-energy-needs-and-what-to-do-abo ut-them/fulltext, Erişim: 22.12.2020.

[8] Elsayed, N., Maida, A. S. ve Bayoumi, M., A Review of Quantum Computer Energy Efficiency, IEEE Green Technologies Conference, 2019.

[9] Grumbling, E. ve Horowitz, M., Quantum Computing: Progress and Prospects, National Academies Press, 2018 
[10] Almudever, C. G., The engineering challenges in quantum computing, IEEE Design, Automation \& Test in Europe Conference \& Exhibition (DATE), 2017.

[11] Upadhyay G. ve Nene, M. J., One time pad generation using quantum superposition states, IEEE International Conference on Recent Trends in Electronics, Information and Communication Technology, 2017.

[12] Li, T. ve Yin, Z., Quantum superposition, entanglement, and state teleportation of a microorganism on an electromechanical oscillator, Science Bulletin, cilt 61, no. 2, pp. 163-171, 2016.

[13] Theurer, T., Resource theory of superposition, Physical review letters, cilt 119, no. 23, 2017.

[14] Yetis, H. ve Karakose, M., Performance Comparison of Population-Based QuantumInspired Evolutionary Algorithms, IEEE 1st International Informatics and Software Engineering Conference (UBMYK), 2019.

[15] Panchi, L. I. ve Zhao, Y., Model and algorithm of sequence-based quantum-inspired neural networks, Chinese Journal of Electronics, 27(1), 9-18, 2018.

[16] Liu, W., Quantum searchable encryption for cloud data based on full-blind quantum computation, Access, 2019.

[17] $\mathrm{Yu}, \mathrm{Y}$., A nested tensor product model transformation, IEEE Transactions on Fuzzy Systems, 27(1), 1-15, 2018.

[18] Adachi, S. H. ve Henderson, M. P., Application of quantum annealing to training of deep neural networks, arXiv preprint arXiv:1510.06356, 2015.

[19] Crawford, D., Levit, A., Ghadermarzy, N., Oberoi, J. S. ve Ronagh, P., Reinforcement learning using quantum boltzmann machines, arXiv preprint:1612.05695, 2016.

[20] Hauke, P. vd., Perspectives of quantum annealing: Methods and implementations, Reports on Progress in Physics, cilt 83, no. 5, 2020.

[21] Liu, J. vd., Adiabatic quantum computation applied to deep learning networks, Entropy, 20(5), 1-28, 2018.

[22] Singh, J. ve Singh, M., Evolution in Quantum Computing, Proceedings of the 5th International Conference on System Modeling and
Advancement in Research Trends (SMART), 2016.

[23] Zhang, G., Quantum-inspired evolutionary algorithms: a survey and empirical study, Journal of Heuristics, cilt 17, no. 3, pp. 303-351, 2011.

[24] Karmakar, S., Dey, A. ve Saha, I., Use of quantum-inspired metaheuristics during last two decades, 7th International Conference on Communication Systems and Network Technologies, 2017.

[25] Patel, O. P. vd., A novel quantum-inspired fuzzy based neural network for data classification, IEEE Transactions on Emerging Topics in Computing, Erken Erişim.

[26] Kerenidis, I. ve Prakash, A., Quantum recommendation systems, arXiv preprint arXiv:1603.08675, 2016.

[27] Tang, E., A quantum-inspired classical algorithm for recommendation systems, 51st Annual ACM SIGACT Symposium on Theory of Computing, 2019.

[28] Panchi, L. ve Ya, Z., Model and algorithm of sequence-based quantum-inspired neural networks, Chinese Journal of Electronics, cilt 27, no. 1, pp. 9-18., 2018.

[29] Wiebe, N., Kapoor, A. ve Svore, K. M., Quantum deep learning, arXiv preprint arXiv:1412.3489, 2014.

[30] Wiebe N. vd., Quantum inspired training for Boltzmann machines, arXiv preprint arXiv:1507.02642, 2015.

[31] Zhou, S., Qingcai, C. ve Xiaolong, W., Deep quantum networks for classification, IEEE 20th International Conference on Pattern Recognition, 2010.

[32] Zhang, Y. ve Ni, Q. Recent advances in quantum machine learning, Quantum Engineering, cilt 2, no. $1,2020$.

[33] Lahoz-Beltra, R., Quantum genetic algorithms for computer scientists, Computers, cilt 5 , no. 4 , 2016.

[34] Potok, T. E. vd., A study of complex deep learning networks on high-performance, neuromorphic, and quantum computers, ACM Journal on Emerging Technologies in Computing Systems (JETC), cilt 14, no. 2, pp. 1-21, 2018. 
[35] Dunjko, V., Taylor, J. M. ve Briegel, H. J., Quantum-enhanced machine learning, Physical review letters, cilt 117, no. 13, 2016.

[36] Benedetti, M., Realpe-Gómez, J. ve PerdomoOrtiz, A., Quantum-assisted helmholtz machines: a quantum-classical deep learning framework for industrial datasets in near-term devices, Quantum Science and Technology, cilt 3, no. 3, 2018.

[37] NASA QuAIL, NASA Quantum Artificial Intelligence Laboratory, Url: https://ti.arc.nasa. gov/tech/dash/groups/quail/, Erişim Tarihi: 22.12.2020.

[38] da Silva, A. J., Ludermir, T. B. ve de Oliveira, W. R., Quantum perceptron over a field and neural network architecture selection in a quantum computer, Neural Networks, cilt 76, pp. 55-64, 2016.

[39] Schuld, M., Sinayskiy, I. ve Petruccione, F., Simulating a perceptron on a quantum computer, Physics Letters, Section A: General, Atomic and Solid State Physics, cilt 379, no. 7, p. 660-66, 2015.

[40] Tacchino, F., Macchiavello, C., Gerace, D. ve Bajoni, D., An artificial neuron implemented on an actual quantum processor, Npj Quantum Information, 5(1), 1-8, 2019.

[41] Steinbrecher, G. R., Quantum optical neural networks, npj Quantum Information, cilt 5, no. 1, pp. 1-9, 2019.

[42] Amin, M. H., Quantum boltzmann machine, Physical Review X, cilt 8, no. 2, 2018.

[43] Nguyen, N., Thuy, T. ve Kenyon, G., Comparing deep learning with quantum inference on the D-Wave 2X, 3rd International Workshop On Post-Moore's Era Supercomputing (PMES), 2018.

[44] Farhi, E. ve Neven, H., Classification with quantum neural networks on near term processors, arXiv preprint arXiv:1802.06002, 2018.

[45] Benedetti, M., Quantum-assisted learning of hardware-embedded probabilistic graphical models, Physical Review X , cilt 7, no. 4, 2017.

[46] Wiebe, N., Kapoor, A. ve Svore, K. M., Quantum deep learning, arXiv preprint arXiv:1412.3489, 2014.

[47] Sagheer, A. ve Zidan, M., Autonomous quantum perceptron neural network, arXiv preprint arXiv:1312.4149, 2013.
[48] Verdon, G., Pye J., Broughton, M., A universal training algorithm for quantum deep learning, arXiv preprint arXiv:1806.09729, 2018.

[49] Kerenidis, I., Landman, J. ve Prakash, A., Quantum algorithms for deep convolutional neural networks, arXiv preprint arXiv:1911.01117,2019. 